\title{
The Effects of Human Bone Marrow-Derived Mesenchymal Stem Cell Conditioned Media Produced with Fetal Bovine Serum or Human Platelet Lysate on Skin Rejuvenation Characteristics
}

\author{
Si-Na Kim ${ }^{1}$, Chan-Ju Lee ${ }^{1}$, JeeHoon Nam ${ }^{1}$, Byeol Choi ${ }^{1}$, Eunkyung Chung ${ }^{1}$, Sun Uk Song ${ }^{1,2}$ \\ ${ }^{I}$ Research Institute, SCM Lifesciences Co. Ltd., Incheon, Korea \\ ${ }^{2}$ Department of Biomedical Sciences, Inha University College of Medicine, Incheon, Korea
}

\begin{abstract}
Background and Objectives: Human mesenchymal stem cell-conditioned medium (MSC-CM) is produced using mesenchymal stem cell culture technology and has various benefits for the skin, including wrinkle removal, skin regeneration, and increased antioxidant activity. Its popularity is thus increasing in the field of functional cosmetics.

Methods and Results: In this study, we analyzed the effects of fetal bovine serum-supplemented MSC-CM (FBSMSC-CM) and human platelet lysate-supplemented MSC-CM (hPL-MSC-CM) on skin rejuvenation characteristics. We found that the concentrations of important growth factors (VEGF, TGF- $\beta 1$, and HGF) and secretory proteins for skin regeneration were significantly higher in hPL-MSC-CM than in FBS-MSC-CM. Furthermore, the capacity for inducing proliferation of human dermal fibroblast (HDF) and keratinocytes, the migration ability of HDF, extracellular matrix (ECM) production such as collagen and elastin was higher in hPL-MSC-CM than that in FBSMSC-CM.

Conclusions: These results support the usefulness and high economic value of hPL-MSC-CM as an alternative source of FBS-MSC-CM in the cosmetic industry for skin rejuvenation.
\end{abstract}

Keywords: Human mesenchymal stem cell, Skin rejuvenation, Fetal bovine serum, Human platelet lysate

\section{Introduction}

The pursuit of modern beauty is often correlated with

Received: April 25, 2020, Revised: October 4, 2020,

Accepted: October 14, 2020, Published online: December 31, 2020 Correspondence to Sun Uk Song

Department of Biomedical Sciences, Inha University College of Medicine, B-308, 366 Seohae-daero, Jung-gu, Incheon 22332, Korea Tel: +82-32-890-2460, Fax: +82-32-891-2277

E-mail: sunuksong@inha.ac.kr

(a) This is an open-access article distributed under the terms of the Creative Commons Attribution Non-Commercial License (http://creativecommons.org/ licenses/by-nc/4.0/), which permits unrestricted non-commercial use, distribution, and reproduction in any medium, provided the original work is properly cited.

Copyright (c) 2021 by the Korean Society for Stem Cell Research mental and physical health. Regardless of age or gender, humans gain self-confidence and contentment by the maintenance of beautiful and healthy skin. As a result, there is increasing research on the development of cosmetic ingredients with pharmacological effects to reflect the desires of an aging society. The goal is to develop functional cosmetics that delay the deterioration of skin functions due to aging, or restore/slow the damage to skin due to aging (1).

Recently, regenerative, stem cell-derived cosmetic products to solve various skin changes due to aging are emerging $(1,2)$. These products are produced using stem cell culture technology and involve several processes. Human mesenchymal stem cells (hMSCs) undergo a process called starvation using serum free culture medium where various 
proteins are secreted from stem cells through intercellular interactions. This raw material is called hMSC-conditioned medium, or MSC-CM $(1,2)$. Proteins secreted from these stem cells including growth factors, cytokines, and extracellular matrix (ECM), are known to rejuvenate the skin in various ways. Growth factors trigger skin regeneration and anti-aging properties, increase elasticity, skin components, hydration, and smooth wrinkles. Similarly, cytokines are effective wound healing and ECM formation and remodeling, known to increase intercellular skin adhesion and enhance skin elasticity $(1,3)$. Human mesenchymal stem cells are the most promising tools available for use in cell therapy and regeneration (4); however, more information needs to be uncovered in order to use them practically $(4,5)$.

For hMSC expansion, fetal bovine serum (FBS) has long been the standard media supplement. However, the risk of zoonotic infection and unclear characterization of its composition cause regulatory authorities to discourage its use for hMSC expansion. As a substitute, many studies have determined the applicability of human platelet lysate (hPL) for hMSC expansion $(5,6)$. Previous studies show that hMSCs expanded under hPL culture conditions have a smaller cell size $(5,7)$ and higher colony forming units-fibroblast (CFU-F) activity, indicating increased stemness compared to those under FBS culture conditions (8). Furthermore, when hMSCs were expanded under hPL culture conditions, their immunophenotype, immunemodulation ability, and differentiation potential were similar, however their ability to proliferate was higher than that of cells cultured in FBS, resulting in more cells being available for manufacture (5).

To develop MSC-CM as a cosmetic raw material, it is prepared after culturing stem cells in FBS-containing culture medium, followed by a starvation process (serum-free condition), hereafter referred to as FBS-MSC-CM. In this study, we applied hPL to MSC culture medium and analyzed the resulting characteristics and function of MSC-CM. To compare this with FBS-MSC-CM, we cultured hMSCs in hPL-containing culture medium, hereafter referred to as hPL-MSC-CM, followed by the starvation process. To date, there has been increasing research on the culture of hMSCs from various sources such as adipose-derived (9) and umbilical cord blood stem cells. However, to the best of our knowledge, no studies have explored the effects of hPL-MSC-CM, especially human bone marrow-derived mesenchymal stem cells (hBMMSCs) or compared the profiles of proteins secreted from FBS-supplemented and hPL-supplemented MSC-CM on the skin.
In this study, we cultured FBS-MSC-CM and hPLMSC-CM using hBM-MSCs and analyzed their effects on skin rejuvenation. These results will provide a foundation for the future research and development of cosmeceuticals based on MSC-CM for more effective skin rejuvenation.

\section{Materials and Methods}

\section{Cell culture}

For the isolation and culture of hMSCs, we used a sub-fractionation culturing method (SCM) as described previously (10). Human BM aspiration for MSC isolation was approved by the Seoul St. Mary's Hospital Institutional Review Board (IRB number \#KC15CSSE0336) and written informed consent was obtained from healthy donors. For culturing human dermal fibroblast (HDF) and keratinocytes, cells were obtained from American Type Cell Collection (ATCC PCS-201-012 for HDF and ATCC PCS-200-011 for keratinocyte, respectively; ATCC, Virginia, US). Cells were grown in DMEM (Thermo Fisher Scientific, 11885-084; Massachusetts, US) supplement with 10\% FBS (Thermo Fisher Scientific) and 1\% penicillin-streptomycin (Thermo Fisher Scientific).

\section{Preparation of FBS-MSC-CM and hPL-MSC-CM}

hMSCs were cultured in phenol red-free-DMEM containing $10 \%$ FBS or $4 \%$ hPL. When the cell confluency reached 80 to $90 \%$, the culture flask was rinsed three times with Dulbecco's Phosphate Buffered Saline (DPBS). Subsequently, $20 \mathrm{ml}$ of phenol red-free, choline chloride-free, serum free medium was added. After $72 \mathrm{~h}$, the conditioned medium (CM) obtained after culturing in FBS-containing medium and in hPL-containing medium hereafter defined as FBS-MSC-CM and hPL-MSC-CM, respectively, were harvested. They were centrifuged at 1500 $\mathrm{rpm}$ for $5 \mathrm{~min}$ then the supernatant was filtered through a $0.22 \mu \mathrm{m}$ filter and stored at $4^{\circ} \mathrm{C}$ until further analysis.

\section{Measurement of growth factor levels in FBS-MSC-CM and hPL-MSC-CM (HGF, VEGF, and TGF- $\beta \mathbf{l}$ )}

The concentration of major growth factors (VEGF, TGF- $\beta 1$, and HGF) in MSC-CM was quantitatively analyzed by ELISA. The Human VEGF Quantikine (R\&D SYSTEMS, SVE00; Minnesota, US), Human TGF- $\beta 1$ Quantikine (R\&D SYSTEMS, SB100B), Human HGF Quantikine Kits (R\&D SYSTEMS, SHG00) were used for analyses according to the manufacturer's instructions. The optical density of the samples was measured using a microplate reader (Biotek, Synergy H1; Vermont, US). 


\section{Human antibody array}

The expression of 105 cytokines was recorded using the Human XL cytokine Array kit (R\&D Systems, ARY022B) according to the manufacturer's instructions. The membrane was detected using a chemiluminescence imager (GE Healthcare, Image Quant LAS 4000 Min; Illinois, US) and the mean pixel density of secreted proteins from hMSCs was analyzed using image J (National Institutes of Health, Maryland, US).

\section{Proliferation assay}

To determine the effects of FBS-MSC-CM and hPLMSC-CM on human dermal fibroblast (HDF) and keratinocyte proliferation, $\mathrm{HDF}$ and keratinocytes were treated with 50\% and 100\% FBS-MSC-CM and hPL-MSC-CM for $24 \mathrm{~h}$. HDF and keratinocyte proliferation were measured using a CCK-8 kit (Cell Counting Kit-8, Dojindo; Maryland, US) according to manufacturer's instructions.

\section{Determination of collagen and elastin content}

Collagen and elastin production of $\mathrm{HDF}$ was measured to compare the effects of MSC-CM on skin elasticity. The Sircol soluble collagen assay (Biocolor, S1000) and Fastin Elastin assay kits (Biocolor, F2000) were used. HDF were cultured in a 6-well culture plate and treated with MSC$\mathrm{CM}$ for $72 \mathrm{~h}$. After performing the assays according to the manufacturer's instructions, collagen and elastin concentrations were measured using a microplate reader (Biotek, Synergy H1).

\section{Determination of MMP-1 and MMP-2 expression}

MMP-1 and MMP-2 expression were analyzed in HDF subjected to UVA irradiation with $168 \mathrm{~mJ} / \mathrm{cm}^{2}$ by a UV lamp (model TN-4LC, K-ACE, Korea) by real-time reverse transcription-polymerase chain reaction (RT-PCR). During irradiation, control cells were prepared equally without exposure of UVA irradiation in the presence or absence of MSC-CM for $72 \mathrm{~h}$. The administration of ascorbic acid (50 $\mu \mathrm{g} / \mathrm{ml}$ ) for $72 \mathrm{~h}$ was applied to positive control for reduction of MMP-1 and MMP-2 expression. Isolated mRNA from cells with RNeasy Mini Kit were used for amplification with TaqMan reverse transcription reagents (Applied Biosystems, California, US) and GAPDH were used for normalized control.

\section{Wound healing assays}

Scratch assays were performed to evaluate the effects of MSC-CM on the migration ability of HDF. In each well of the 24-well culture plate containing wound inserts, $1 \times 10^{5} \mathrm{HDF}$ were seeded. After the cells formed a mono- layer with the wound, FBS-MSC-CM or hPL-MSC-CM were treated. After $6 \mathrm{~h}$, the wells were fixed and stained with $10 \%$ formaldehyde and $1 \%$ crystal violet solution, respectively, and images of the wound healing process were captured. For quantification, the width $(\mathrm{cm})$ of the wound was measured to compare the wound healing levels of cells cultured in different conditioned medium.

\section{Statistical analysis}

All results are presented as mean \pm standard deviation (SD). One-way ANOVA was used to compare the treatment groups and a p-value $<0.05$ was considered statistically significant. Statistical tests were conducted using GraphPad Prism 6.0 software (Graphpad; California, US).

\section{Results}

\section{$\mathrm{hPL}-\mathrm{MSC}-\mathrm{CM}$ is richer in secretory proteins than FBS-MSC-CM}

First, we explored whether the expression of secreted proteins varies in the two different culture media (FBSsupplement vs. hPL-supplement). We identified a higher number of proteins in hPL-MSC-CM $(n=39)$ than in FBS-MSC-CM ( $\mathrm{n}=25$ ) (Fig 1A). More specifically, 25 proteins were identified in both MSC-CMs, whereas 14 proteins were identified only in the hPL-MSC-CM (Fig. 1B). We found that the concentrations of 11 proteins, Angiopoietin-1, Chitinase 3-like 1, Cystatin C, IL-17A, IL-22, IGFBP-3, MCP-1, MIF, Pentraxin3, Serpin E1, VCAM-1, were significantly higher in both FBS-MSC-CM and hPL-MSC-CM than in the control medium, but there was no significant difference in proteins between FBSMSC-CM and hPL-MSC-CM (Fig. 1C). All 9 proteins, except Cystatin C and IL-17A, are reported to be involved in skin cell proliferation, skin-repairing angiogenesis, and wound healing (11-17). Cystatin C and IL-17A were reported to regulate antibacterial activity in skin $(18,19)$.

The 14 proteins, angiogenin, complement factor $\mathrm{D}$, Dkk-1, endoglin, emmprin, FGF-7, FGF-19, HGF, IGFBP-2, osteopontin, SDF- $\alpha$ 1, Thrombospondin-1, uPAR, and VEGF, were significantly upregulated in hPL-MSCCM comparing with FBS-MSC-CM. Among these, 13 proteins except complement factor D are involved in skin rejuvenation processes such as wound healing, skin cell growth, skin regeneration, and ECM remodeling (20-29). However, to the best of our knowledge, there is no report on the direct effect of complement factor D on the skin, known to involved in the alternative pathway of the complement system (Fig. 1D). Importantly, 14 proteins, adiponectin, apolipoprotein $\mathrm{A}-1, \mathrm{BDNF}, \mathrm{C} 5 / \mathrm{C} 5 \mathrm{a}, \mathrm{C}$ reactive 

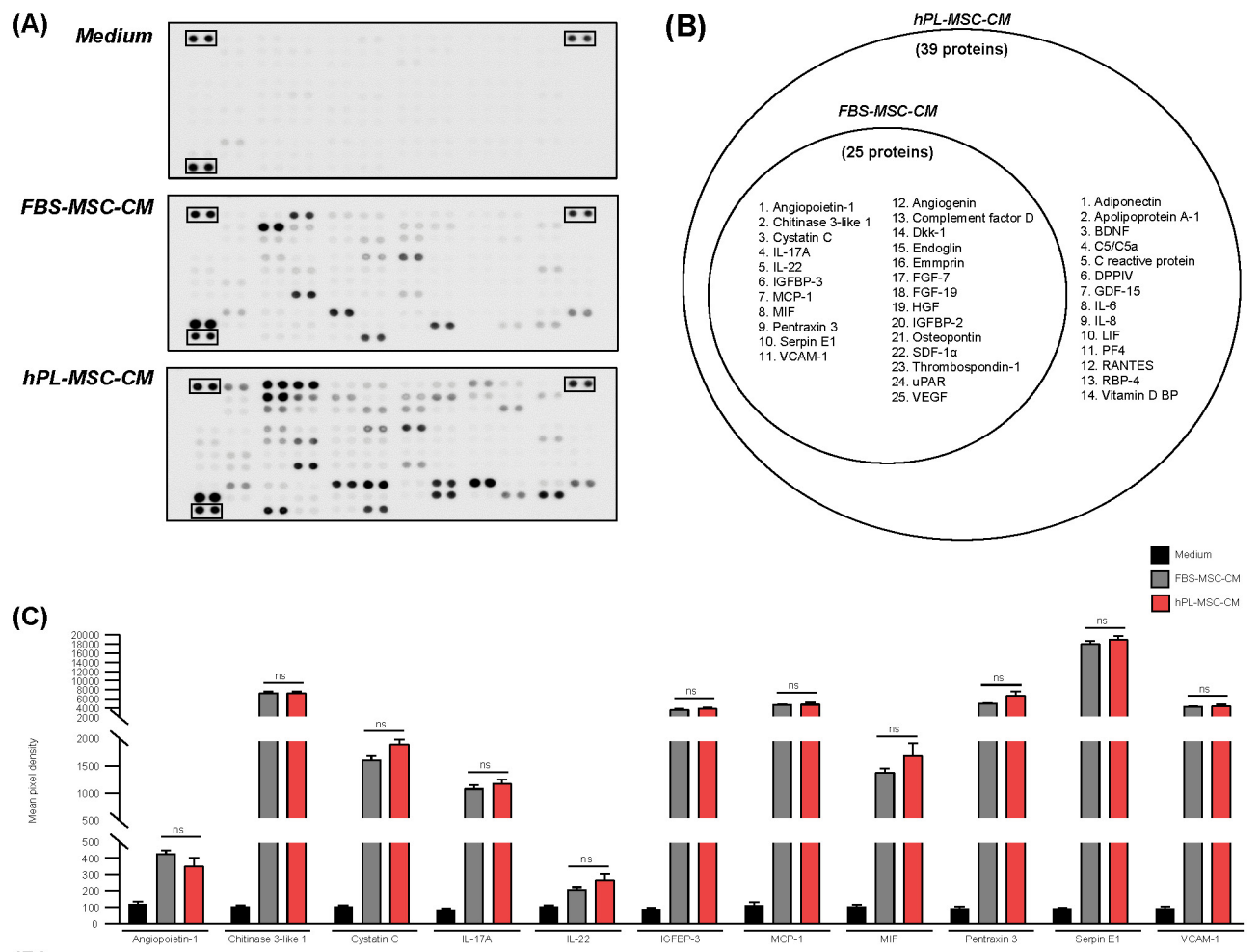

(D)

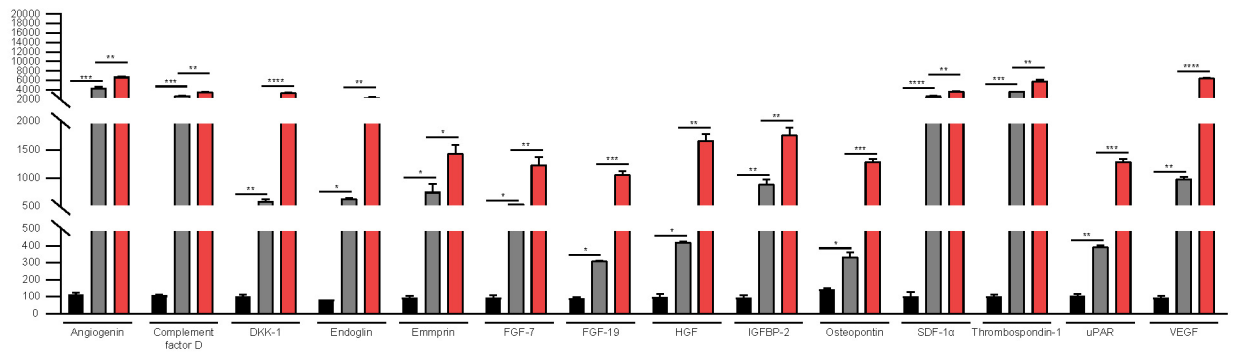

(E)

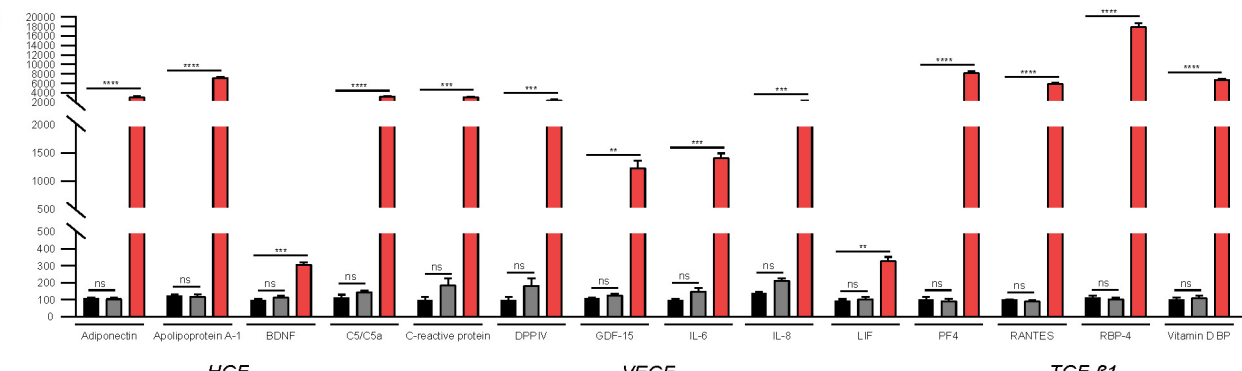

(F)
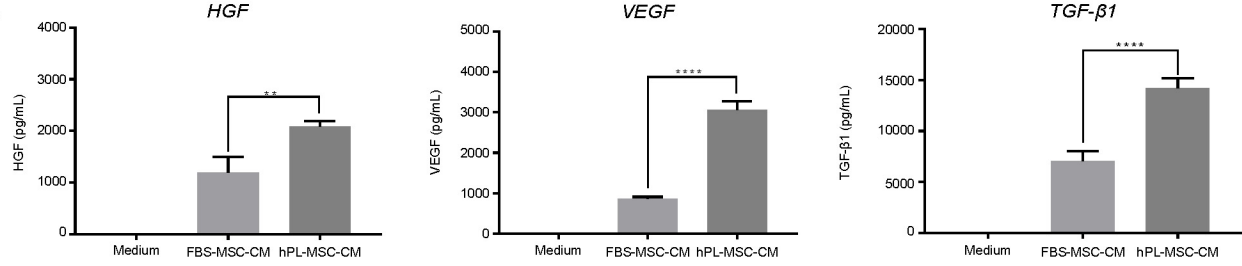

Fig. 1. Profiling comparison of secretory proteins in FBS-MSC-CM and hPL-MSC-CM. (A) Analysis of proteome profiler human XL cytokines in FBS-MSC-CM and hPL-MSC-CM were determined in parallel. Reference spots are indicated by square boxes on the membrane blot. (B) Classification of secreted proteins detected in FBS-MSC-CM and hPL-MSC-CM by the relative analysis of pixel density using image J, we plotted the proteins detected in both MSC-CMs and in hPL-MSC-CM alone. (C) Secretory proteins detected in FBS-MSC-CM and hPL-MSC-CM. (D) Secretory proteins detected at a higher concentration in hPL-MSC-CM. (E) Secretory proteins detected in hPL-MSC-CM alone. (F) Concentrations of HGF, VEGF, and TGF- $\beta 1$ in both MSC-CMs; HGF, VEGF, and TGF- $\beta 1$ were analyzed quantitatively by ELISA in FBS-MSC-CM and hPL-MSC-CM. Data are represented as mean \pm SD. $*<0.05, * *<0.01, * * *<0.001, * * *<0.0001$. 
(A)

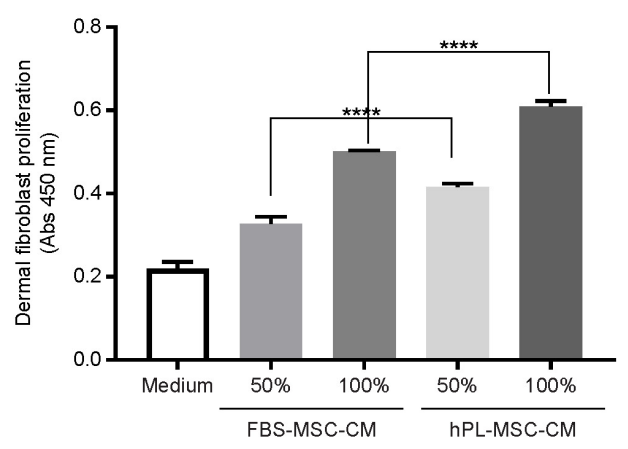

(B)

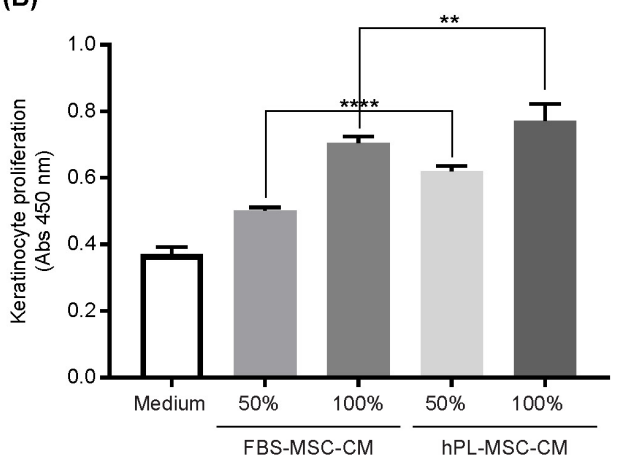

Fig. 2. Effect of FBS-MSC-CM and hPL-MSC-CM on proliferation of human skin cells. (A) Proliferation ability following treatment of human dermal fibroblasts with both MSCCMs for $24 \mathrm{~h}$ using CCK-8. (B) Proliferation ability of keratinocytes. Data are represented as mean \pm SD. $* *<0.01, * * * *<0.0001$. protein, DPPIV, GDF-15, IL-6, IL-8, LIF, PF4, RANTES, RBP-4, and Vitamin D BP, were detected only in hPLMSC-CM, but not in FBS-MSC-CM (Fig. 1E). Among them, 6 proteins, Adiponectin, BDNF, DPPIV, PF4, RANTES, and RBP-4, are involved in skin regeneration (30-35), whereas 3 proteins, IL-6, IL-8, and Vitamin D BP, play a role in reducing skin inflammation $(36,37)$. IL-6 is also known to stimulate collagen production in $\mathrm{HDF}$ (38). However, the rest 5 proteins, apolipoprotein A-1, C5/C5a, C-reactive protein, GDF-15, and LIF, are not associated with skin rejuvenation. The protein array map and full name of the cytokine arrays from the array kit used in this experiment are summarized in the supplementary data (Supplementary Table S1).

In addition, we quantified representative growth HGF, VEGF, and TGF- $\beta 1$ that enhanced skin regeneration and reduced skin inflammation. We found that all the three growth factors were significantly higher in hPL-MSC-CM than in FBS-MSC-CM (Fig. 1F) indicating that hPL culture conditions are more advantageous than FBS in increasing the concentrations and numbers of skin-related proteins secreted from hMSCs.

\section{$\mathrm{hPL}-\mathrm{MSC}-\mathrm{CM}$ is more potent than FBS-MSC-CM in stimulating skin cells growth}

Next, we conducted experiments to determine whether hPL-MSC-CM, which is rich in skin-related proteins, has better efficacy in skin improvement. Increased cell proliferation of HDF improves the regeneration of damaged skin and increased cell proliferation of keratinocytes strengthens the skin barrier by increasing intercellular adhesion (1). We compared the effects of FBS-MSC-CM and hPL-MSC-CM on the proliferation of skin cells, HDF and keratinocytes.

We found that $\mathrm{HDF}$ proliferation was significantly higher in hPL-MSC-CM than in FBS-MSC-CM (Fig. 2A). Similarly, hPL-MSC-CM demonstrated superior efficacy on keratinocyte proliferation compared with FBS-MSCCM (Fig. 2B). Thus, hPL-MSC-CM stimulates proliferation of skin cells more effectively than FBS-MSC-CM expecting better efficacy in skin regeneration and rejuvenation.

\section{$\mathrm{hPL}-\mathrm{MSC}-\mathrm{CM}$ is more potent than FBS-MSC-CM in accelerating HDF migration}

A previous study showed that increased proliferation and migration of $\mathrm{HDF}$ suggests enhanced skin regeneration (1). Accordingly, to determine the effect of MSC-CM on migration of HDFs, a wound scratch assay was performed. Both FBS-MSC-CM and hPL-MSC-CM increased the migration of $\mathrm{HDF}$ compared to control, without any treatment of conditioned medium, however, The hPL-MSC-CM showed the superior efficacy compared to FBS-MSC-CM (Fig. 3A). In consistent, the wound width $(\mathrm{cm})$ was significantly reduced in both FBS-MSCCM and hPL-MSC-CM compared with control and the superior efficacy of $\mathrm{HDF}$ migration was observed in hPL-MSC-CM (Fig. 3B).

\section{$\mathrm{hPL}-\mathrm{MSC}-\mathrm{CM}$ is more potent in the promotion of $\mathrm{ECM}$ production and remodeling}

To determine the effect of MSC-CM on ECM production and remodeling by HDF, we firstly measured the concentrations of collagen and elastin. We found that the synthesis of collagen and elastin increased in both MSC$\mathrm{CMs}$, however, the increased extent was higher in hPLMSC-CM than FBS-MSC-CM (Fig. 4A). Additionally, the expression levels of MMP-1 and MMP-2 were examined in HDF subjected to UVA irradiation to determine the efficacy in ECM remodeling. In response to UVA, MMP-1 and MMP-2 were induced, which completely blocked by ascorbic acid (AA, $50 \mu \mathrm{g} / \mathrm{ml}$ )) treatment in HDF (Fig. 4B). In the presence of both MSC-CMs, the blockade of MMP-1 induction was observed in similar with AA, more- 

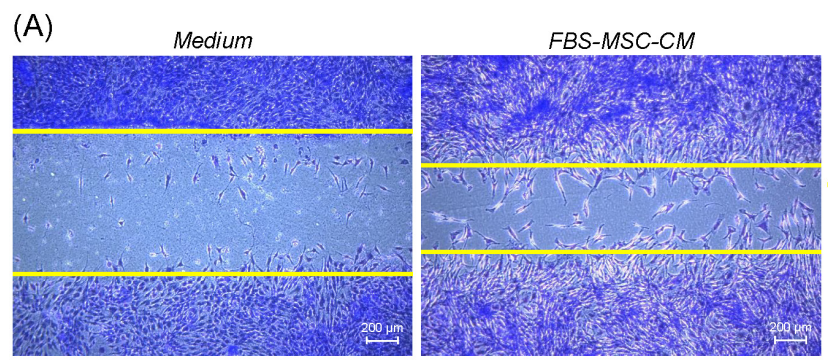

(B)
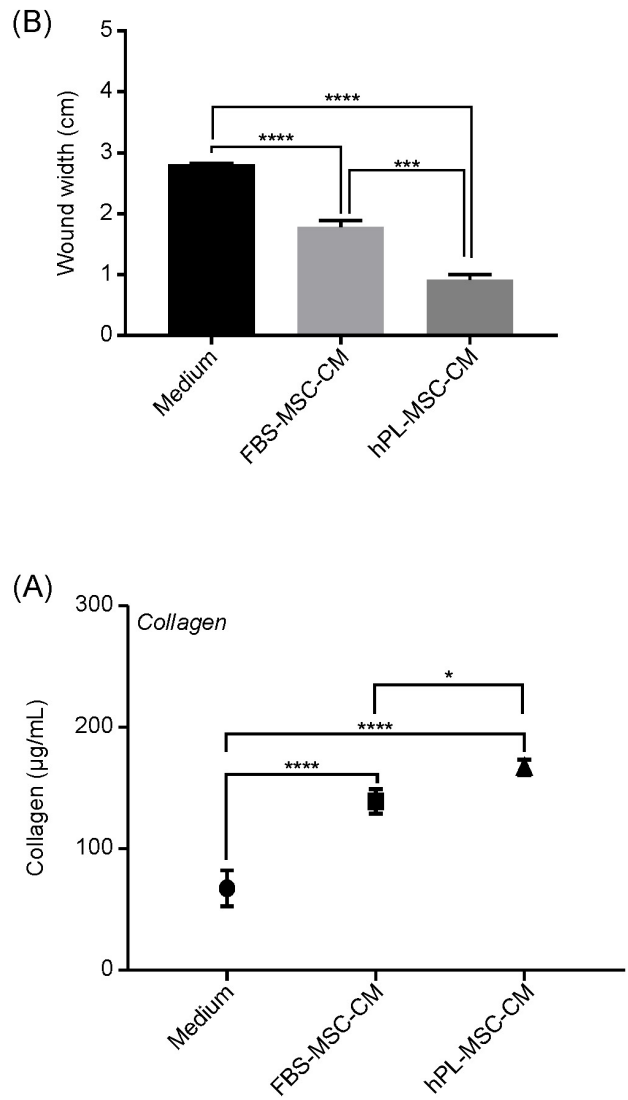

(B)

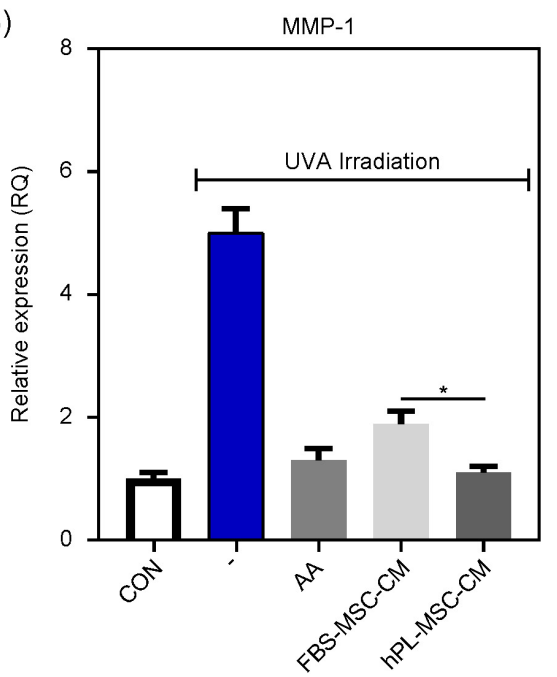

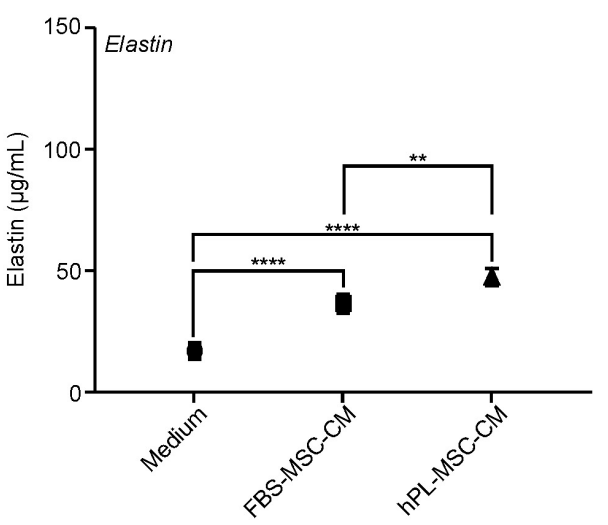

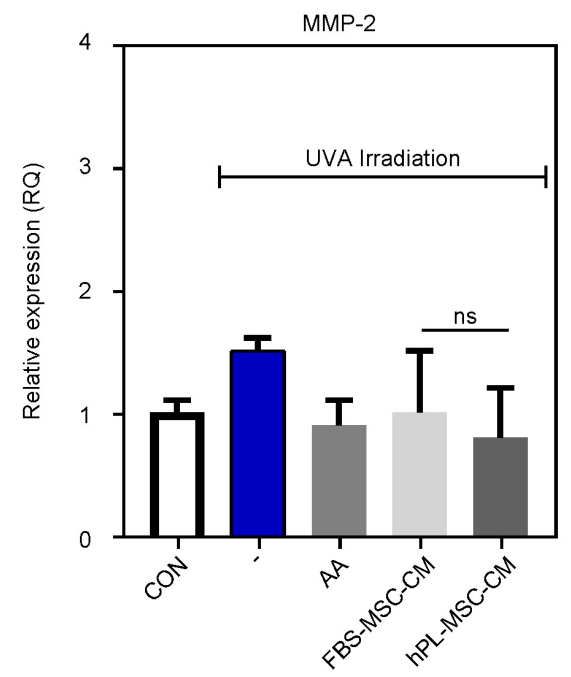

Fig. 3. Effects of FBS-MSC-CM and hPL-MSC-CM on migration ability. (A) A wound was created with a wound insert on human dermal fibroblasts to determine the migration ability of cells (X 40). Serum free media without MSC culture were used as controls (Medium). Scale bar: $200 \mu \mathrm{M}$. (B) Wound width (cm) of Medium, FBS-MSC-CM, and hPL-MSC-CM were measured and quantified.

Fig. 4. Effects of FBS-MSC-CM and hPL-MSC-CM on ECM production and remodeling. (A) The concentrations of collagen and elastin produced by MSC-CM were measured. Data are represented as mean \pm SD. $*<0.05, \quad * *<0.01, \quad * * *<0.001$, $* * * *<0.0001$. (B) After HDF were exposed to UVA (168 mJ/ $\left.\mathrm{cm}^{2}\right)$ using UV lamp, the cells were treated Ascorbic acid (AA, positive control), FBS-MSC-CM and hPL-MSC-CM for 72 hours. UVA-induced MMPs (MMP1, MMP-2) expression was determined by RT-PCR. Value are shown as mean \pm SD. ${ }^{*} p<0.05$ compared with HDF only with UVA irradiation. 
over, hPL-MSC-CM was better than FBS-MSC-CM. However, inhibition of MMP-2 induction was observed with similar extent in both conditions. This suggests that hPL-MSC-CM may be more effective at skin regeneration for ECM production and ECM remodeling via MMP-1 than FBS-MSC-CM.

\section{Discussion}

Human mesenchymal stem cells have self-renewal ability and multi-differentiation capacities and are useful for treating various immune diseases as well as in regenerative medicine $(4,5)$. Recently, in the cosmetic industry, the research and development of cosmetics using stem cells has increased (2). MSC-CMs contain various growth factors and cytokines, and ECM proteins, which are known to promote the regeneration of damaged skin tissues and play an important role in skin rejuvenation (2). In particular, growth factors such as EGF, bFGF, VEGF, and TGF- $\beta 1$, secreted from stem cells play a major role in skin regeneration, anti-aging, ECM production $(1,9)$.

$\mathrm{hPL}$ is one of the most promising substitutes for FBS. There have been many studies evaluating the use of hPL on the ex-vivo expansion of hMSCs, which show that it increases cell proliferation but does not affect the immunophenotype, immune-modulatory potential, or differentiation potential of hMSCs $(7,8)$. Similarly, we found that hPL did not affect the immunophenotype and immune-modulating function of hMSCs, but increased proliferation (data not shown). However, other studies have shown that hMSCs cultured in hPL-supplemented medium have a more homogeneous population in terms of cell size and internal complexity than those cultured in FBS-supplemented medium (7, 8). Similarly, Griffiths et al. (7) showed that hPL-supplemented medium culture of senescent hMSCs demonstrates cellular rejuvenation with decreased cell sizes and population doubling times (PDT). The effect of hPL on MSCs has been shown to enhance proliferation, functionality, and characteristics of MSC in previous studies $(7,8)$.

In this study, we found out that hPL is also effective in improving the quality of MSC-CM as well as the cells. The quality of MSC-CM is related to the number and concentration of secreted proteins. We identified 25 and 39 secreted proteins in FBS-MSC-CM and hPL-MSC-CM, respectively. In addition, we found that the concentration of secretory proteins HGF, VEGF and TGF- $\beta 1$ that enhanced skin regeneration and reduced skin inflammation were significantly higher in hPL-MSC-CM than FBS-
MSC-CM. These results suggest that hPL creates a favorable culture environment for increasing the number and concentration of secreted proteins in manufacturing MSC-CM, which may also improve its functionality.

Adipose-derived stem cells (ADSCs), and Umbilical cord blood (UCB)-derived MSC-CMs are known to affect skin rejuvenation such as by inducing the proliferation and migration or by increasing the production of ECM such as collagen, elastin, and fibronectin. In this study, we found out that hPL-MSC-CM shows more effective performance than FBS-MSC-CM in stimulating HDF and keratinocyte proliferation as well as in promoting the migration ECM production, and MMP-1-mediated ECM remodeling of HDF. Taken together, hPL-MSC-CM has superior efficacy in skin rejuvenation compared to FBSMSC-CM.

Among the proteins detected in hPL-MSC-CM, complement factor D, apolipoprotein A-1, C5 / C5a, C-reactive protein, GDF-15, and LIF are not known to be related to skin rejuvenation and further experiments to determine function of these proteins on the skin are required.

To the best of our knowledge, there are no studies to date on the effects of hPL-MSC-CM on the skin. There have been no analyses or comparisons of the secretome profiles and effects on the skin of stem cells produced under different culture conditions (FBS-supplement $v s$. hPLsupplement). The manufacturing method using hPL enables a human-based and xeno-free culture system containing no animal-derived ingredients in the process of obtaining cosmetic raw materials. We have developed an efficient method to enrich secretory proteins using hPL to produce a xeno-free stem cell culture medium. This process may improve the manufacture of MSC-CM, thus enhancing the overall quality of cosmetics.

\section{Potential Conflict of Interest}

The authors have no conflicting financial interest.

\section{Supplementary Materials}

Supplementary data including one table can be found with this article online at https://doi.org/10.15283/ijsc20070

\section{References}

1. Muthukumar A, Seetharam RN. Stem cell derived cosmetic products: an overview. Manipal J Med Sci 2016;1:46-52

2. Vizoso FJ, Eiro N, Cid S, Schneider J, Perez-Fernandez R. Mesenchymal stem cell secretome: toward cell-free therapeutic strategies in regenerative medicine. Int J Mol Sci 


\section{7; 18:1852}

3. Barrientos S, Brem H, Stojadinovic O, Tomic-Canic M. Clinical application of growth factors and cytokines in wound healing. Wound Repair Regen 2014;22:569-578

4. Van Pham P. Mesenchymal stem cells in clinical applications. In: Van Pham P, editor. Stem Cell Processing. Stem Cells in Clinical Applications. Cham: Springer; 2016. 37-69

5. Becherucci V, Piccini L, Casamassima S, Bisin S, Gori V, Gentile F, Ceccantini R, De Rienzo E, Bindi B, Pavan P, Cunial V, Allegro E, Ermini S, Brugnolo F, Astori G, Bambi F. Human platelet lysate in mesenchymal stromal cell expansion according to a GMP grade protocol: a cell factory experience. Stem Cell Res Ther 2018;9:124

6. Hemeda H, Giebel B, Wagner W. Evaluation of human platelet lysate versus fetal bovine serum for culture of mesenchymal stromal cells. Cytotherapy 2014;16:170-180

7. Griffiths S, Baraniak PR, Copland IB, Nerem RM, McDevitt TC. Human platelet lysate stimulates high-passage and senescent human multipotent mesenchymal stromal cell growth and rejuvenation in vitro. Cytotherapy 2013;15:1469-1483

8. Schallmoser K, Bartmann C, Rohde E, Reinisch A, Kashofer K, Stadelmeyer E, Drexler C, Lanzer G, Linkesch W, Strunk D. Human platelet lysate can replace fetal bovine serum for clinical-scale expansion of functional mesenchymal stromal cells. Transfusion 2007;47:1436-1446

9. Kim WS, Park SH, Ahn SJ, Kim HK, Park JS, Lee GY, Kim KJ, Whang KK, Kang SH, Park BS, Sung JH. Whitening effect of adipose-derived stem cells: a critical role of TGF-beta 1. Biol Pharm Bull 2008;31:606-610

10. Song SU, Kim CS, Yoon SP, Kim SK, Lee MH, Kang JS, Choi GS, Moon SH, Choi MS, Cho YK, Son BK. Variations of clonal marrow stem cell lines established from human bone marrow in surface epitopes, differentiation potential, gene expression, and cytokine secretion. Stem Cells Dev 2008;17:451-461

11. Korpela E, Yohan D, Chin LC, Kim A, Huang X, Sade S, Van Slyke P, Dumont DJ, Liu SK. Vasculotide, an Angiopoietin-1 mimetic, reduces acute skin ionizing radiation damage in a preclinical mouse model. BMC Cancer 2014;14:614

12. Lecron JC, Paris I, Bernard FX, Morel F. Contribution of IL-22 to experimental skin inflammation. In: Quesniaux V, Ryffel B, Padova F, editor. IL-17, IL-22 and Their Producing Cells: Role in Inflammation and Autoimmunity. Basel: Springer Basel; 2013. 305-317

13. Liu J, Guo Y, Huang Y, Xue H, Bai S, Zhu J, Xia X, Shen B, Fang W. Effects of insulin-like growth factor binding protein 3 on apoptosis of cutaneous squamous cell carcinoma cells. Onco Targets Ther 2018;11:6569-6577

14. Shallo H, Plackett TP, Heinrich SA, Kovacs EJ. Monocyte chemoattractant protein-1 (MCP-1) and macrophage infiltration into the skin after burn injury in aged mice. Burns 2003;29:641-647

15. Shimizu T. Role of macrophage migration inhibitory factor
(MIF) in the skin. J Dermatol Sci 2005;37:65-73

16. Cappuzzello C, Doni A, Dander E, Pasqualini F, Nebuloni M, Bottazzi B, Mantovani A, Biondi A, Garlanda C, D'Amico G. Mesenchymal stromal cell-derived PTX3 promotes wound healing via fibrin remodeling. J Invest Dermatol 2016;136:293-300

17. Simone TM, Longmate WM, Law BK, Higgins PJ. Targeted inhibition of PAI-1 activity impairs epithelial migration and wound closure following cutaneous injury. Adv Wound Care (New Rochelle) 2015;4:321-328

18. Archer NK, Adappa ND, Palmer JN, Cohen NA, Harro JM, Lee SK, Miller LS, Shirtliff ME. Interleukin-17A (IL-17A) and IL-17F are critical for antimicrobial peptide production and Clearance of Staphylococcus aureus nasal colonization. Infect Immun 2016;84:3575-3583

19. Blankenvoorde MF, van't Hof W, Walgreen-Weterings E, van Steenbergen TJ, Brand HS, Veerman EC, Nieuw Amerongen AV. Cystatin and cystatin-derived peptides have antibacterial activity against the pathogen Porphyromonas gingivalis. Biol Chem 1998;379:1371-1375

20. Yamaguchi Y, Morita A, Maeda A, Hearing VJ. Regulation of skin pigmentation and thickness by Dickkopf 1 (DKK1). J Investig Dermatol Symp Proc 2009;14:73-75

21. Valluru M, Staton CA, Reed MW, Brown NJ. Transforming growth factor- $\beta$ and endoglin signaling orchestrate wound healing. Front Physiol 2011;2:89

22. Huet E, Vallée B, Szul D, Verrecchia F, Mourah S, Jester JV, Hoang-Xuan T, Menashi S, Gabison EE. Extracellular matrix metalloproteinase inducer/CD147 promotes myofibroblast differentiation by inducing alpha-smooth muscle actin expression and collagen gel contraction: implications in tissue remodeling. FASEB J 2008;22:1144-1154

23. Nunes QM, Li Y, Sun C, Kinnunen TK, Fernig DG. Fibroblast growth factors as tissue repair and regeneration therapeutics. PeerJ 2016;4:e1535

24. Shim JH, Park JY, Lee MG, Kang HH, Lee TR, Shin DW. Human dermal stem/progenitor cell-derived conditioned medium ameliorates ultraviolet a-induced damage of normal human dermal fibroblasts. PLoS One 2013;8:e67604

25. Guo R, Chai L, Chen L, Chen W, Ge L, Li X, Li H, Li S, Cao C. Stromal cell-derived factor 1 (SDF-1) accelerated skin wound healing by promoting the migration and proliferation of epidermal stem cells. In Vitro Cell Dev Biol Anim 2015;51:578-585

26. Weber CE, Li NY, Wai PY, Kuo PC. Epithelial-mesenchymal transition, TGF- $\beta$, and osteopontin in wound healing and tissue remodeling after injury. J Burn Care Res 2012;33:311-318

27. Detmar M. The role of VEGF and thrombospondins in skin angiogenesis. J Dermatol Sci 2000;24(Suppl 1):S78-S84

28. Gulyaev A, Piven V. Rat model of healing the skin wounds and joint inflammations by recombinant human angiogenin, erythropoietin and tumor necrosis factor- $\alpha$. In: Osman NAA, Abas WABW, Wahab AKA, Ting HN, editor. 5th Kuala Lumpur International Conference on Biomedical Engineering 2011. IFMBE Proceedings, vol 35. Berlin, 
Heidelberg: Springer; 2011. 854-857

29. D'Alessio S, Gerasi L, Blasi F. uPAR-deficient mouse keratinocytes fail to produce EGFR-dependent laminin-5, affecting migration in vivo and in vitro. J Cell Sci 2008; 121(Pt 23):3922-3932

30. Shibata S, Tada Y, Asano Y, Hau CS, Kato T, Saeki H, Yamauchi T, Kubota N, Kadowaki T, Sato S. Adiponectin regulates cutaneous wound healing by promoting keratinocyte proliferation and migration via the ERK signaling pathway. J Immunol 2012;189:3231-3241

31. Palazzo E, Marconi A, Truzzi F, Dallaglio K, Petrachi T, Humbert P, Schnebert S, Perrier E, Dumas M, Pincelli C. Role of neurotrophins on dermal fibroblast survival and differentiation. J Cell Physiol 2012;227:1017-1025

32. Eisman R, Surrey S, Ramachandran B, Schwartz E, Poncz M. Structural and functional comparison of the genes for human platelet factor 4 and PF4alt. Blood 1990;76:336-344

33. van den Broek LJ, Kroeze KL, Waaijman T, Breetveld M, Sampat-Sardjoepersad SC, Niessen FB, Middelkoop E, Scheper RJ, Gibbs S. Differential response of human adipose tissue-derived mesenchymal stem cells, dermal fibro- blasts, and keratinocytes to burn wound exudates: potential role of skin-specific chemokine CCL27. Tissue Eng Part A 2014;20:197-209

34. Stephensen CB. Vitamin A, infection, and immune function. Annu Rev Nutr 2001;21:167-192

35. Rennekampff HO, Hansbrough JF, Kiessig V, Doré C, Sticherling M, Schröder JM. Bioactive interleukin-8 is expressed in wounds and enhances wound healing. J Surg Res 2000;93:41-54

36. Gorman S, Geldenhuys S, Judge M, Weeden CE, Waithman J, Hart PH. Dietary vitamin D increases percentages and function of regulatory $\mathrm{T}$ cells in the skin-draining lymph nodes and suppresses dermal inflammation. J Immunol Res 2016;2016:1426503

37. Lee EG, Mickle-Kawar BM, Gallucci RM. IL-6 deficiency exacerbates skin inflammation in a murine model of irritant dermatitis. J Immunotoxicol 2013;10:192-200

38. Duncan MR, Berman B. Stimulation of collagen and glycosaminoglycan production in cultured human adult dermal fibroblasts by recombinant human interleukin 6. J Invest Dermatol 1991;97:686-692 